The Egyptian Journal of Hospital Medicine (April 2019) Vol. 75 (3), Page 2418-2425

\title{
Two-Dimensional and Three-Dimensional Echocardiographic Assessment of Aortic Root Diameter in Patients with Coronary Ectasia
}

\author{
Mohammed Hesham Hassan Ezzat, Mohammed Adel Attia, Islam Ibrahim Ahmed Hassan Abualwafa* \\ Department of Cardiology, Faculty of Medicine (Damietta), Al-Azhar University, Egypt \\ *Corresponding author: Islam Ibrahim Ahmed Hassan Abualwafa, Mobile: (+20) 01025252217, \\ E-Mail: ehab_abualwafa8878@yahoo.com
}

\begin{abstract}
Background: Coronary artery ectasia (CAE) is a well-recognized but relatively uncommon finding encountered during diagnostic coronary angiography. It is commonly defined as in appropriate dilation of the coronary arteries exceeding the largest diameter of an adjacent normal vessel more than 1.5-fold. CAE is not an isolated and benign disease but a reflection of a generalized vascular media defect.

Objective: The aim of this study was to compare 2D-TTEand 3D-TTE measurements of the aortic root diameter in patients with coronary artery ectasia to assess the presence of aortic root dilatation.

Patients and methods: This prospective observational study included 50 consecutive patients came to the Department of Cardiology, Al-Azhar University Hospital, New Damietta for coronary angiography. The study was carried out from November 2017 until December 2018. Injection aortography was used as a gold standard and to assess the presence of ascending aorta dilatation in those patients.

Results: The present study shows that there was a good correlation between 3D-TTE and aortography at the levels of aortic annulus, sinuses of Valsalva, sinotubular junction $(\mathrm{r}=0.98,0.95,0.98)$ but a rough correlation between 2D-TTE and aortography at these levels $(r=0.49,0.48,0.46)$.The present study shows that there was increase prevalence of aortic root dilatation 13 patients (26\%) and ascending aorta dilatation 9patients(18\%) in patients with CAE .

Conclusions: Accuracy of aortic root measurement by 3DTTE was superior to that by 2DTTE, because the values by 2DTTE were underestimated compared to those measured by 3DTTE and aortography. Increase prevalence of aortic root dilatation and ascending aorta dilatation in patients with coronary artery ectasia. Dilated Ascending aorta was associated with a higher prevalence of aortic root dilatation.
\end{abstract}

Keywords: Two Dimensional, Three Dimensional Echocardiographic, CAE.

\section{INTRODUCTION}

Coronary artery dilations, including coronary ectasia and aneurysms, are a subgroup of coronary artery anomalies and one of the most neglected topics in cardiovascular medicine ${ }^{(\mathbf{1})}$.

Coronary artery ectasia is a wellrecognized but relatively uncommon finding encountered during diagnostic coronary angiography ${ }^{(2)}$. Although discovered incidentally, they can cause detrimental events such as sudden cardiac death ${ }^{(3)}$.

Coronary artery ectasia represents not only an anatomical variant but also a clinical constellation of coronary artery disease like association with myocardial ischemia and acute coronary syndromes. Coronary dilatations are thus classified as anomalies of intrinsic coronary arterial anatomy and have been reported in up to $5 \%$ of retrospective studies ${ }^{(4)}$.

They can occur either as a diffuse dilation of the coronary arteries $(>1.5$ times the normal diameter) that involves $50 \%$ of the artery length,

which is called "ectasia" or as a localized dilation in which the aneurysmal dilation of the largest coronary vessel by 1.5 times is seen in $<50 \%$ of the total vessel length. Coronary artery disease is considered the underlying factor in most of these patients ${ }^{(5)}$.

Some coronary dilatations have been described in association with inflammatory connective tissue disorders such as scleroderma or Kawasaki disease or with a history of cocaine abuse ${ }^{(\boldsymbol{6})}$.

The term "aortic root" refers to the aortic valve from its position at the left ventricular outlet to its junction with the ascending portion of the aorta (7).

It is the direct continuation of the left ventricular outflow tract. Aortoventricular junction refers to the junction between the left ventricular structures and the aortic valvularsinuses, this representing the anatomic junction, or the semilunar lines of attachment of the arterial valvular leaflets, this locus representing the hemodynamic ventriculoarterial junction ${ }^{\left({ }^{8}\right)}$.

CAE is not an isolated and benign disease but a reflection of a generalized vascular media defect. Very few studies have reported a higher incidence of coronary dilatations in patients with aneurysms of the abdominal aorta and its branches (9).

The aim of this study was to compare 2DTTEand 3D-TTE measurements of the aortic root diameter in patients with coronary artery ectasia to assess the presence of aortic root dilatation. 


\section{PATIENTS AND METHODS}

This prospective observational study included a total of 50 consecutive patients attending at Department of Cardiology, Al-Azhar University Hospital, New Damietta for coronary angiography. Approval of the ethical committee and a written informed consent from all the subjects were obtained. This study was conducted between November 2017 until December 2018.

the study was designed to compare 2D-TTE and 3DTTE measurements of the aortic root diameter in patients with coronary artery ectasia. Injection aortography was used as a gold standard and to assess the presence of ascending aortic dilatation in those patients.

Inclusion Criteria: Patients who are admitted for coronary angiography according to guidelines indications and showed presence of coronary ectasia with or without coronary artery occlusion.

Exclusion criteria: Moderate to severe valvular heart disease affecting aortic root diameter and if there was a contraindication of coronary angiography.

The age of the study population ranged from $41-83$ years. All patients were subjected to clinical history and physical examination with emphasis on age, gender, medical history of diabetes mellitus (10), hypertension ${ }^{(1)}$, dyslipidemia ${ }^{(12)}$, smoking ${ }^{(13)}$, previous medications and associated comorbidity.

Coronary angiography was done according to guideline indications and if there was diffuse coronary ectasia, injection aortography was performed to measure the diameter of aortic root at the level of aortic annulus, aortic sinuses of valsalva and aortic sinotubular junction and to measure the diameter of ascending aorta to assess the presence of ectasia of the ascending Aorta. Boles et al defined $\mathrm{CAE}$ as an arterial segment with a diameter at least 1.5 times the diameter of the adjacent normal coronary artery ${ }^{(\mathbf{1 4})}$.

\section{Transthoracic Echocardiography}

Patients underwent 2D TTE and 3D TTE at echocardiogrhy laboratory of the Cardiology Department at Al-Azhar University Hospital New Damietta, using Philips IE 33 (Philips Ultrasound, 22100 Bothell Everett Highway, Bothell, WA, 98021 USA). The probe used were the X 5-1 and $X$ 7-1 phased array-sector probe (frequency range 1.5-4.3 MHz).

The image data were stored in the hard disk and were transferred to a computer for off-line analysis obtained by a piece of analysis software $(\mathrm{Q}-$
Lab 10). Images of the long axis of the left ventricle in the parasternal view and while the $3 \mathrm{D}$ zoomed image of the long axis and short axis of the aortic root in the parasternal view was recorded in the full -volume mode for four consecutive beats.

\section{- Measurement of aortic root diameters by 2D and} 3D echocardiography

Tow dimensional transthoracic
echocardiography:

Standard diameter measurements are at the aortic annulus, at the level of the sinuses of Valsalva and at the sinotubular junction (Figure 1).

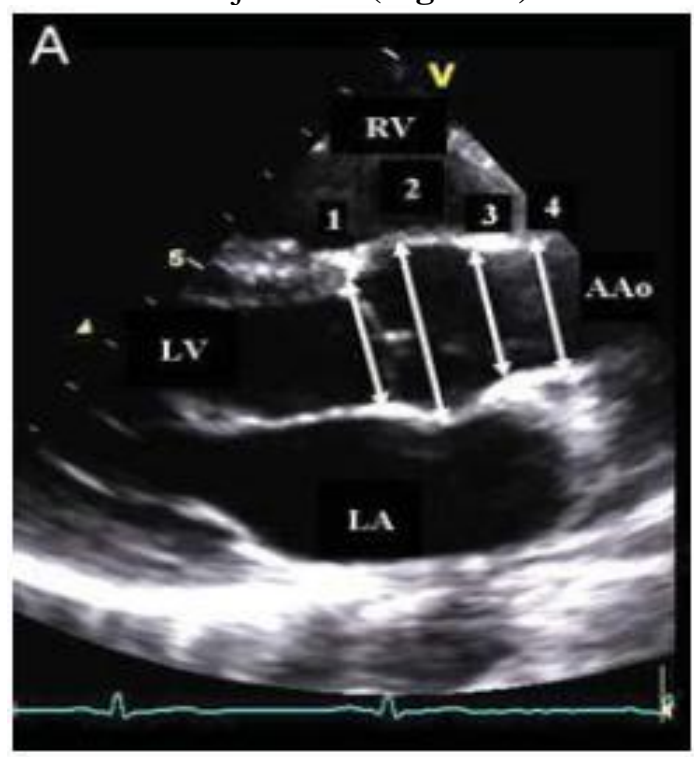

Figure 1: The following diameters are shown: aortic annulus diameter (1), sinuses of Valsalva (2), sinotubular junction (3), and tubular ascending aorta (4) ${ }^{(15)}$.

The aortic annulus diameter was measured by 2D TTE from the para sternal long-axis view using the zoomed images at the insertion of the leaflets at the end of diastole perpendicular to the long axis of the aorta from leading edge to leading edge. The spaces between the luminal surface of the three bulges on the aortic root and their respective valvular leaflets are known as the aortic sinuses of Valsalva ${ }^{(\mathbf{1 6})}$.

The sinuses of valsalva is the largest diameter of aortic root. The sinotubular junction is defined as the top of the sinuses of valsalva and is recognized by the acute angle at the transition from the curved sinuses to the tubular ascending aorta ${ }^{(16)}$.

Three dimensional trans thoracic echocardiography:

The diameters of the aortic root by 3DTTE were measured at para sternal short axis view using full volume, multiple cut planes will be obtained by I- slice modality at the level of aortic annulus, aortic sinuses of valsalva and sinotubular junction, two dimensions will be measured( longitudinal width, vertical ), obtained by a piece of analysis software (Q-Lab 10). 


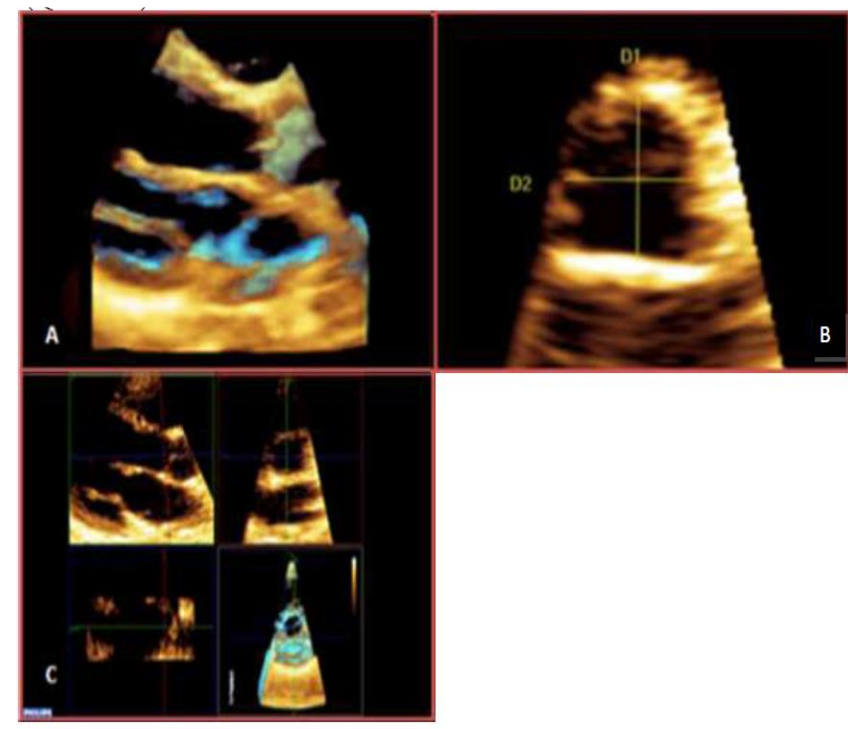

Figure 2: Measurement of aortic annulus diameters in real-time three-dimensional (3D) echocardiography $\mathbf{A}$. Para sternal long-axis view in real-time 3D echocardiography.B. Horizontal(D2) and vertical (D1) diameters measured from the short-axis view using zoom mode after multiplanar review C. Multi planar review mode of the $3 \mathrm{D}$ live acquisition with the three orthogonal cutting plans at baseline: in the top left window, one plane (blue line) is parallel to the long axis of the ascending aorta; a second plane (red line) is perpendicular to the previous line and set at the origin of the cusp to define the level of the aortic annulus; in the left bottom window, the third plane (green plane) is perpendicular to the two others; in the top right window, a short-axis view of the aortic annulus ${ }^{(17)}$.

\section{Normal values of aortic segments}

The normal values of the various aortic segments are shown in (Figure 3).

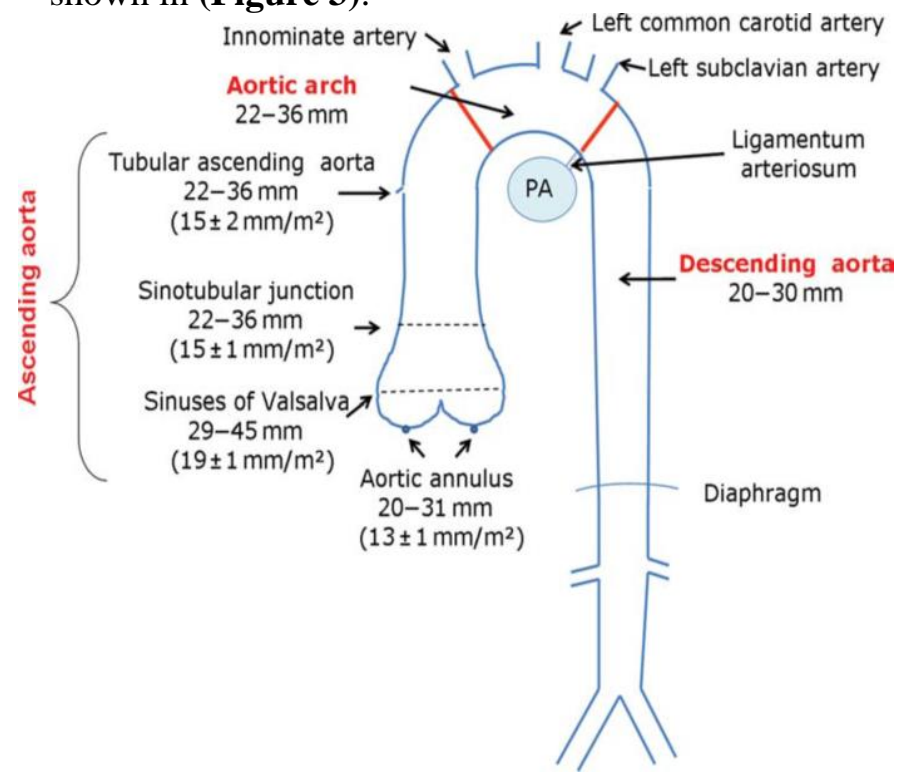

Figure 3: Normal size of thoracic aortic segments. The thoracic aorta can be divided into three segments: the ascending aorta that extends from the aortic annulus to the innominate artery and is typically measured at the level of the aortic annulus, the sinuses of Valsalva, the sinotubular junction, and the proximal (tubular) ascending aorta; the aortic arch that extends from the innominate artery to the ligamentum arteriosum; and the descending aorta that extends from the ligamentum arteriosum to the level of the diaphragm. PA, right pulmonary artery (18).

\section{Statistical analysis}

All demographic, clinical, and technical data were collected and tabulated using the "Data Collection Form" and entered into a computerized database. Statistical Package for Social Sciences (SPSS) version 15 for Windows ${ }^{\circledR}$ (SPSS Inc, Chicago, IL, USA) was used for analyses. The data were summarized using descriptive statistics: mean, standard deviation, minimal and maximum values for quantitative variables and number and percentage for qualitative values. Correlations were done to test for linear relations between variables . P value was considered significant if $<0.05$.

\section{RESULTS}

Demographic and clinical data:

This study included 50 patients, 34 of them $(68 \%)$ were males, while 16 (32\%) were females; their ages ranged between 41 years and 83 years with (mean $\pm \mathrm{SD}=59.02 \pm 8.56$ years). $26(52 \%)$ were hypertensive, 21(42\%) were diabetics, 19 (38\%) were smokers and $15(30 \%)$ were dyslipidemia (table 1).

Table 1: Showing the characteristics of the patient

\begin{tabular}{|c|c|c|}
\hline Variable & Number & Percentage \\
\hline Male & 34 & $68 \%$ \\
\hline Female & 16 & $32 \%$ \\
\hline DM & 21 & $42 \%$ \\
\hline No DM & 29 & $58 \%$ \\
\hline HTN & 26 & $52 \%$ \\
\hline No HTN & 24 & $48 \%$ \\
\hline Smoker & 19 & $38 \%$ \\
\hline Nonsmoker & 31 & $62 \%$ \\
\hline Dyslipdemic & 15 & $30 \%$ \\
\hline Not dyslipidemic & 35 & $70 \%$ \\
\hline
\end{tabular}

Tables (2) and (3) show comparisons and correlation between diameter of aortic annulus in 2D, vertical diameter in 3D and diameter of aortic annulus in aortography. 
Table 2: Comparisons and correlation between diameter of aortic annulus in 2D and vertical diameter in 3D

\begin{tabular}{|l|l|c|c|c|}
\hline Variable & Mean & SD & P & R \\
\hline 2D & 2.3 & 0.34 & & \\
3D & 2.8 & 0.48 & 0.002 & 0.49 \\
\hline
\end{tabular}

Table 3: Comparisons and correlation between diameter of aortic annulus in 2D and diameter of aortic annulus in aortography

\begin{tabular}{|l|l|l|l|l|}
\hline Variable & Mean & SD & P & R \\
\hline 2D & 2.3 & 0.34 & & \\
\hline Aortography & 2.8 & 0.51 & 0.002 & 0.49 \\
\hline
\end{tabular}

Table 4: Comparisons and correlation between vertical diameter of aortic annulus in $3 \mathrm{D}$ and diameter of aortic annulus in aortography

\begin{tabular}{|l|l|c|c|c|}
\hline Variable & Mean & SD & P & R \\
\hline 3D & 2.8 & 0.48 & & \\
\hline Aortography & 2.8 & 0.51 & 0.001 & 0.98 \\
\hline
\end{tabular}

The tables (2-4) showing that there were statistically significant difference between diameter of aortic annulus in 2D, 3D and aortography but a good correlation $(r=0.98)$ was observed between the values obtained by 3DTTE and aortography ; however, the correlation between the values by 2DTTE and aortography was rough $(r=0.49)$ j

\section{Comparisons and correlation between diameter of sinuses of valsalva in 2D, vertical diameter in 3D and diameter of aortic sinuses in aortography.}

Table 5: Comparisons and correlation between diameter of aortic sinuses of valsalva in $2 \mathrm{D}$ and vertical diameter in 3D

\begin{tabular}{|l|l|l|l|c|}
\hline Variable & Mean & SD & P & R \\
\hline 2D & 3.2 & 0.30 & & \\
\hline 3D & 3.80 & 0.68 & 0.01 & 0.43 \\
\hline
\end{tabular}

Table 6: Comparisons and correlation between diameter of sinuses of valsalva in $2 \mathrm{D}$ and between diameter of aortic sinuses of valsalva in aortography

\begin{tabular}{|l|l|l|l|l|}
\hline Variable & Mean & SD & P & R \\
\hline 2D & 3.2 & 0.30 & & \\
\hline Aortography & 3.8 & 0.69 & 0.002 & 0.48 \\
\hline
\end{tabular}

Table 7 : Comparisons and correlation between vertical diameter of sinuses of valsalva in 3D anddiameter of aortic sinuses in aortography .

\begin{tabular}{|l|l|r|c|c|}
\hline Variable & Mean & SD & P & R \\
\hline 3D & 3.8 & 0.68 & & \\
\hline Aortography & 3.8 & 0.69 & 0.001 & 0.95 \\
\hline
\end{tabular}

The tables (5-7)showing that there were statistically significant difference between diameter of aortic sinuses in 2D, 3D and aortographybut A good correlation $(\mathrm{r}=0.95)$ was observed between the values obtained by 3DTTE and aortography ; however, the correlation between the values by 2DTTE and aortography was weak $(r=0.48)$

Comparisons and correlation between diameter of sinotubular junction in 2D, vertical diameter in 3D and diameter of sinotubular junction in aortography.

Table 8: Comparisons and correlation between diameter of sinotubular junction in 2D and vertical diameter in 3D .

\begin{tabular}{|l|l|l|c|c|}
\hline Variable & Mean & SD & P & R \\
\hline 2D & 2.8 & 0.26 & & \\
\hline 3D & 3.2 & 0.61 & 0.002 & 0.47 \\
\hline
\end{tabular}

Table 9: Comparisons and correlation between diameter of sinotubular junction in 2D and between diameter of sinotubular junction in aortography .

\begin{tabular}{|l|l|c|c|c|}
\hline Variable & Mean & SD & P & R \\
\hline 2D & 2.8 & 0.26 & & \\
\hline Aortography & 3.2 & 0.63 & 0.001 & 0.46 \\
\hline
\end{tabular}

Table 10: Comparisons and correlation between vertical diameter of sinotubular junction in 3D and diameter of sinotubular junction in aortography .

\begin{tabular}{|l|c|c|c|c|}
\hline Variable & Mean & SD & P & R \\
\hline 3D & 3.2 & 0.61 & & \\
\hline Aortography & 3.2 & 0.63 & 0.001 & 0.98 \\
\hline
\end{tabular}

The tables (8-10)showing that there were statistically significant difference between diameter of sinotubular junction in 2D, 3D and aortography but $\mathrm{A}$ good correlation $(\mathrm{r}=0.98)$ was observed between the values obtained by 3DTTE and aortography ; however, the correlation between the values by 2DTTE and aortography was weak $(r=0.46)$.

Prevalence of dilated aortic root diameter in patients with coronary artery ectasia.

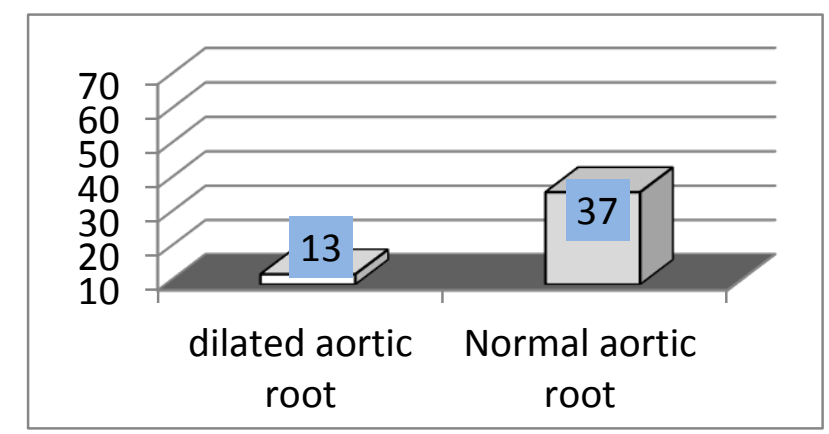

Fig .4. Diagram showing number of patients with dilated aortic root diameter and with normal aortic root diameter in the study population . 
Prevalence of dilated Ascending aorta diameter in patients with coronary artery ectasia

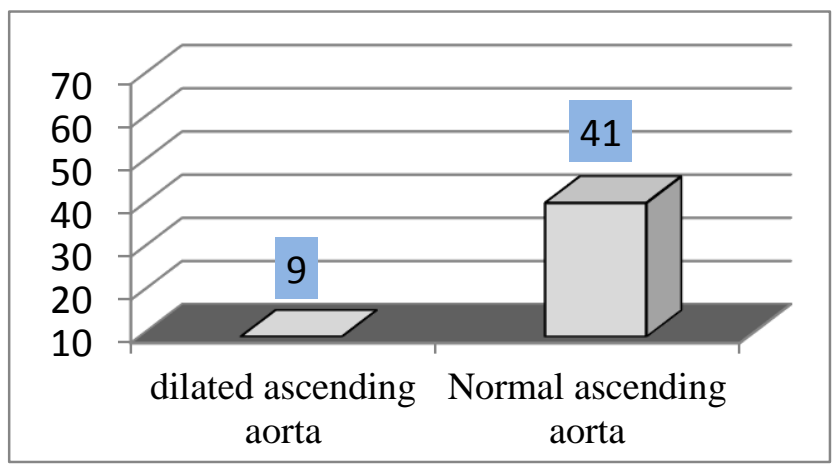

Fig 5. Diagram showing number of patients with dilated ascending aorta diameter and with normal ascending aorta diameter in the study population. Comparisons and correlation between vertical and longitudinal diameter of $3 \mathrm{D}$ Aortic annulus

In the present study the diameter of aortic annulus in 3D vertical mean was $(2.8 \pm 0.48)$ $\mathrm{cm}$, and in 3D longitudinal mean was $(2.8 \pm$ $0.46) \mathrm{cm}$. There was a good correlation 1 between them.

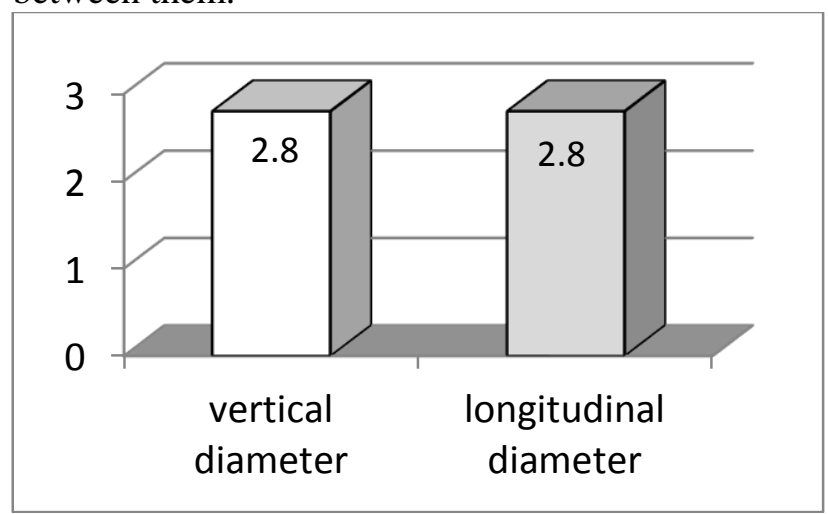

Fig 6. Diagram showing Comparisons and correlation between vertical and longitudinal diameter of 3 D Aortic annulus

\section{Comparisons and correlation between} vertical and longitudinal diameter of $3 \mathrm{D}$ Aortic sinuses of valsalva

The diameter of sinuses of valsalvain 3D vertical mean was $(3.8 \pm 0.68) \mathrm{cm}$ and in $3 \mathrm{D}$ longitudinal mean was $(3.7 \pm 0.66) \mathrm{cm}$. There was a good correlation between them .

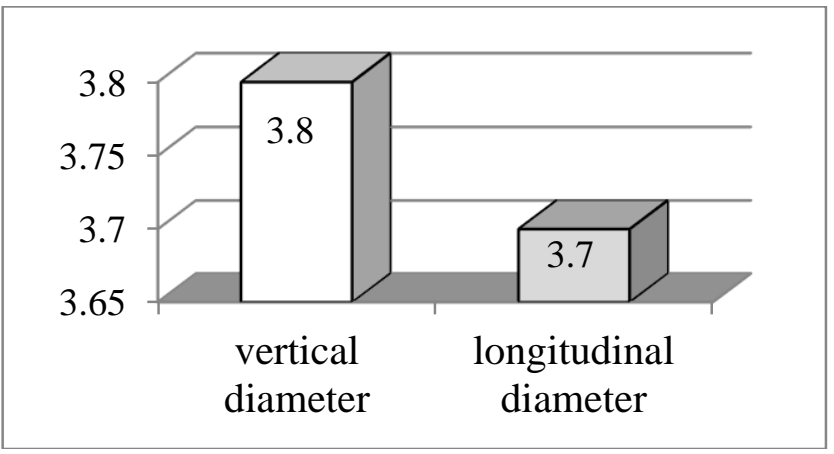

Fig 7. Diagram showing Comparisons and correlation between vertical and longitudinal diameter of 3 D Aortic sinuses of Valsalva.

Comparisons and correlation between vertical and longitudinal diameter of $3 \mathrm{D}$ Aortic sinotubular junction

The diameter of sinuses of Valsalva in 3D vertical mean was $(3.2 \pm 0.61) \mathrm{cm}$ and in $3 \mathrm{D}$ longitudinal mean was $(3.1 \pm 0.60) \mathrm{cm}$. There was a good correlation between them.

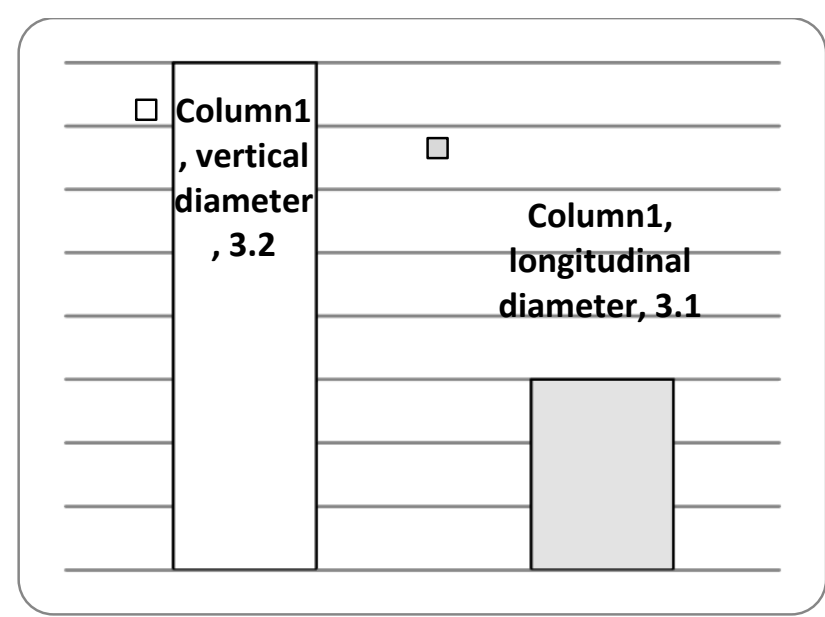

Fig. 8. Diagram showing Comparisons and correlation between vertical and longitudinal diameter of 3 D Aortic sinotubular junction

\section{DISCUSSION}

Coronary artery ectasia has been associated with aneurysms or dilatations in other vascular beds, so that patients with CAE may have a generalized dilatation diathesis related or not with atherosclerosis ${ }^{(\mathbf{1 9})}$.

The present study was designed to compare 2D-TTEand 3D-TTE measurements of the aortic root diameter in patients with coronary artery ectasia to assess the presence of aortic root dilatation. Injection aortography was used as a gold standard and to assess the presence of ascending aortic dilatation in those patients.

This prospective observational study included 50 patients with coronary ectasia. Patients with moderate to severe valvular heart disease affecting aortic root diameter and those with contra indications of coronary angiography were excluded. 
All patients were subjected to routine clinical history and physical examination then Patients underwent 2DTTE and 3DTTE at echocardiography laboratory and the data collected from the echo were compared with the data collected from of Aortography.

In the present work the age of studied cases was ranged between 41 years and 83 years with mean $59.02 \pm 8.56$ years. In contrary to these results, Kahraman et al. ${ }^{(20)}$ reported that mean age was 57 \pm 11 year. This discrepancy may be attributed to different inclusion criteria and variation in population and the number of included cases in each study as their work was done on 80 patients with $\mathrm{CAE}$ and 25 control patients.

In the present study there were 50 patients 34 of them (68\%) were males, while $16(32 \%)$ were females, $26(52 \%)$ were hypertensive, $21(42 \%)$ were DM, 19 (38\%) were smokers , 15 (30\%) were dyslipidemia. In contradiction to the results of the present study Kahraman et al. ${ }^{(20)}$ reported that males were $40(50 \%)$ in the CAE group, 48 (60\%) were HTN, $12(15 \%)$ were DM, $26(32 \%)$ were smokers and $33(41 \%)$ were dyslipidemia. This discrepancy in results may be attributed to different inclusion and exclusion criteria as they excluded patients with wall motion abnormality, cardiomyopathy prior myocardial infarction, also they excluded patients with connective tissue or inflammatory diseases.

In the present study there is increase number of patients with dilated Ascending aorta diameter as there are 9 patients (18\%) with dilated ascending aorta. This prevalence of dilated ascending aorta is nearly seven times as the prevalence in the study of Benedetti and Hope ${ }^{(21)}$, where 671 patients (2.7\%) Of the 24,992 patients included in the study undergoing routine chest CT examinations had reported dilatation of the ascending aorta.

The results of this study also are somewhat similar to the study of Hatemi et al. ${ }^{(22)}$ where the ascending aortic diameter (AAD) was larger in both the coronary artery aneurysm (CAA) and coronary dilatation (CD) groups than in patients lacking CAAs and CDs.

On the contrary, in the study of Triantafyllidi et al. (23) both ascending aortic diameter and ascending Aortic index were significantly increased in patients with CAE compared with patients with normal coronary arteries. Moreover, these results are also confirmed by another study Palomares $\boldsymbol{e t}$ al. ${ }^{\text {(24) }}$ in which it was reported that patients operated for ascending aorta aneurysm have a fivefold increase in the frequency of angiographically detected CAE compared with a concomitantly studied cohort of patients with suspected CAD.
Those findings additional to results from previous studies, concluded that coronary ectasia is not an isolated and benign disease but a reflection of a generalized vascular media defect ${ }^{(25)}$.

In the present study there is increase number of patients with dilated aortic root as there are 13 patients $(26 \%)$ with dilated aortic root at the sinuses of valsalva level while the prevalence of dilated aortic root is $4 \%$ in the general population as mentioned in the Alegret et al. ${ }^{(26)}$ where they reported that dilated aortic root is a relatively common finding, with a prevalence of about $4 \%$ measured at the level of the sinuses of Valsalva . This means that in patients with coronary artery ectasia the prevalence of dilated aortic root is more than six times as the general population.

In the current study, it was observed that a dilated Ascending aorta was associated with a higher prevalence of dilatation of annulus, sinuses of valsalva and sinotubularjunction. The prevalence was $77.7 \%$ (7/9) for both annulus and sinotubular junction, and $88.8 \%$ (8/9) for sinuses of valsalva. In contradiction with the results of the present study Ocak et al. ${ }^{(27)}$ reported that in patients with dilated Ascending aorta $38 \%$ had annulus dilation, $52 \%$ had a dilated sinuses of valsalva, and $21 \%$ had sinotubular junction dilatation, compared to $28 \%$, $28 \%$, and $4 \%$ for annulus, sinuses of valsalva and sinotubular junction dilatation respectively in patients with a normal ascending aorta. This discrepancy in results may be attributed to different inclusion and exclusion criteria because they included patients with high suspicion of aortic aneurysm and/or dissection and exclude all patients with coexistent proximal aortic pathology or surgery, Patients with Marfan syndrome and bicuspid aortic valve .

In the present study the diameter of aortic annulus in $2 \mathrm{D}$ echo mean was $(2.30 \pm 0.34) \mathrm{cm}$. Those results are somewhat similar to several recent studies such as Callaghan et al. ${ }^{(28)}$ where mean was $(2.13 \pm 0.23) \mathrm{cm}$. In the present study the diameter of aortic annulus in $3 \mathrm{D}$ echo mean was $(2.80 \pm 0.48) \mathrm{cm}$. In contradiction with the results of the present study (28). The mean was $(2.07 \pm 0.26) \mathrm{cm}$. This discrepancy in results may be attributed to that the previous study doesn't exclude patient with structural heart disease as it contain $9 \%$ of the patient was with valvular heart disease and 5\% was diagnosed as cardiomyopathy.

The present study shows that there were statistically significant difference between diameter of aortic annulus in 2D, 3D and aortography, but a good correlation ( $r=0.98)$ was observed between the values obtained by 3DTTE and aortography ; however, the correlation between the values by 2DTTE and aortography was rough $(\mathrm{r}=0.49)$ 
Regarding the diameters of aortic sinuses of valsalva in this study, the diameter in $2 \mathrm{D}$ echo mean was $(3.2 \pm 0.30) \mathrm{cm}$ while the diameter in $3 \mathrm{D}$ echo mean was $(3.8 \pm 0.68) \mathrm{cm}$ and the diameter in aortography mean was $(3.8 \pm 0.69) \mathrm{cm}$. Thus, there was statistically significant difference between diameter of aortic sinuses of valsalva in 2D, 3D and aortography, but A good correlation $(r=0.95)$ was observed between the values obtained by 3DTTE and aortography ; however, the correlation between the values by 2DTTE and aortography was weak ( $\mathrm{r}$ $=0.48)$.

As regard the diameter of aortic sinotubular junction in 2D echo mean in the present study, it was $(2.8 \pm 0.26) \mathrm{cm}$ while the diameter in $3 \mathrm{D}$ echo mean was $(3.2 \pm 0.61) \mathrm{cm}$ and the diameter in aortography mean was $(3.2 \pm 0.63) \mathrm{cm}$, so there was statistically significant difference between diameter of sinotubular junction in 2D, 3D and aortography, but A good correlation $(r=0.98)$ was observed between the values obtained by 3DTTE and aortography ; however, the correlation between the values by 2DTTE and aortography was weak $(r=0.46)$.

By comparing the 3D vertical and longitudinal diameter of aortic root at the levels of annulus , sinuses of valsalva and sinotubular junction respectively, it was found that the diameter of aortic annulus in 3D longitudinal mean was $(2.8 \pm 0.46)$ $\mathrm{cm}$ and in 3D vertical mean was $(2.8 \pm 0.48) \mathrm{cm}$ ,while the diameter of sinuses of valsalva in $3 \mathrm{D}$ longitudinal mean was $(3.7 \pm 0.66) \mathrm{cm}$ and in $3 \mathrm{D}$ vertical mean was $(3.8 \pm 0.68) \mathrm{cm}$, while the diameter of sinotubular junction in 3D longitudinal mean was $(3.1 \pm 0.60) \mathrm{cm}$ and in $3 \mathrm{D}$ vertical mean was(3.2 \pm 0.61$) \mathrm{cm}$, thus there was a good correlation between 3D longitudinal and vertical diameters of aortic annulus, sinuses of valsalva and sinotubular junction $(r=0.98,0.97,0.95)$ respectively .

\section{CONCLUSION}

It could be concluded that accuracy of aortic root measurement by 3DTTE was superior to that by 2DTTE, because the values by 2DTTE were underestimated compared to those measured by 3DTTE and aortography. Increase prevalence of aortic root dilatation and ascending aorta dilatation in patients with coronary artery ectasia. Finally, dilated Ascending aorta was associated with a higher prevalence of aortic root dilatation.

\section{RECOMMENDATION}

1. The images obtained by 3DTTE provided accurate values of the aortic root, which were equal to the values measured by aortography. 3DTTE complements the conventional echocardiographic assessment of $\mathrm{AV}$ and aortic root with more accurate and reproducible quantitative analysis of aortic root morphology and can be performed repeatedly and noninvasively unlike the main current methods for evaluating aortic root such as MDCT and 3DTEE.

2. In patients with coronary artery ectasia, there is higher possibility of dilated aortic root and dilated ascending aorta, so we should do injection aortography, 2D and 3D echocardiographic assessment of aortic root at the three levels and ascending aorta for detection of aortic root and/or ascending aorta dilatation ..

\section{REFERENCES}

1. Mosterd WL (2018): Cardiac abnormalities and complications in Ehlers-Danlos syndrome. Ehlers-Danlos Syndrome: A Multidisciplinary Approach. http://ebooks.iospress.com/book/ehlers-danlos-syndromea-multidisciplinary-approach

2. Hayes SN, Kim ES, Saw J et al. (2018): Spontaneous coronary artery dissection: current state of the science: a scientific statement from the American Heart Association. Circulation, 137(19):523-557.

3. Sabet A, Haghighiabyaneh M, Tazelaar $\mathrm{H}$ et al. (2018): The Clinical Dilemma of Cardiac Fibroelastic Papilloma. Structural Heart, 2(4): 274-280.

4. Gallego P3, Rodríguez-Puras MJ, Gotarredona PS et al. (2018): Prevalence and prognostic significance of pulmonary artery aneurysms in adults with congenital heart disease. International Journal of Cardiology, 270:120-125.

5. Bhave NM, Isselbacher EM and Eagle KA (2019): Aortic Aneurysms. In Comprehensive Cardiovascular Medicine in the Primary Care Setting. Humana Press Cham., 6: 365-377.

6. Mavrogeni SI, Dimitroulas T and Kitas GD (2019): Cardiovascular magnetic resonance in the diagnosis and management of cardiac and vascular involvement in the systemic vasculitides. Current Opinion in Rheumatology, 31(1):16-24.

7. Gould RA, Aziz H, Woods CE et al. (2019): ROBO4 variants predispose individuals to bicuspid aortic valve and thoracic aortic aneurysm. Nature Genetics, 51(1): 42-45.

8. McCarthy K, Franklin R, Slavik $Z$ et al. (2019): Simplified Guide to Understanding the Anatomy of Congenital Heart Disease. In Critical Heart Disease in Infants and Children. Elsevier.

9. Stenson KM, Patterson BO, Grima MJ et al. (2019): Midterm results of endovascular aneurysm sealing to treat abdominal aortic aneurysm. Journal of Vascular Surgery, 69(1): 53-62.

10. Harrison LC (2019): Type 1 Diabetes. In Clinical Immunology; Fifth Edition. https://www.elsevier.com/books/clinicalimmunology/9780702068966

11. Vargas-Uricoechea $H$ and Cáceres-Acosta MF (2018): Blood pressure control and impact on cardiovascular events in patients with type 2 diabetes mellitus. Open Med (Wars), 13: 304-323.

12. Al Rifai M, Martin SS, McEvoy JW et al. (2018): The prevalence and correlates of subclinical atherosclerosis among adults with low-density lipoprotein cholesterol< 70 $\mathrm{mg} / \mathrm{dL}$ : The Multi-Ethnic Study of Atherosclerosis (MESA) and Brazilian Longitudinal Study of Adult Health 
Two-Dimensional and Three-Dimensional Echocardiographic Assessment...

(ELSA-Brasil). Atherosclerosis, 274: 61-66.

13. Bhatt SP, Anderson JA, Brook RD et al. (2018): Cigarette smoking and response to inhaled corticosteroids in COPD. European Respiratory Journal, 51(1): 170-173.

14. Boles U, Johansson A, Wiklund U et al. (2018): Cytokine Disturbances in Coronary Artery Ectasia Do Not Support Atherosclerosis Pathogenesis. International Journal of Molecular Sciences, 19(1): 260-166.

15. Goldstein SA, Arturo E, Suhny A et al. (2015): Multimodality imaging of diseases of the thoracic aorta in adults: from the American Society of Echocardiography and the European Association of Cardiovascular Imaging: endorsed by the Society of Cardiovascular Computed Tomography and Society for Cardiovascular Magnetic Resonance. Journal of the American Society of Echocardiography, 2: 119-182.

16. Galea N, Piatti F, Sturla F et al. (2018): Novel insights by 4D Flow imaging on aortic flow physiology after valvesparing root replacement with or without neo sinuses. Interactive Cardiovascular and Thoracic Surgery, 26(6):957-964.

17. Fries RC, Gordon SG, Saunders AB et al. (2019): Quantitative assessment of two-and three-dimensional trans thoracic and two-dimensional trans esophageal echocardiography, computed tomography, and magnetic resonance imaging in normal canine hearts. Journal of Veterinary Cardiology, 21: 79-92.

18. David TE (2019): Aortic valve-sparing operations. In Atlas of Cardiac Surgical Techniques. Korean J Thorac Cardiovasc Surg., 45(4): 205-212.

19. Soto JA, Lucey BC (2016): Emergency Radiology: The Requisites E-Book. Elsevier Health Sciences. https://www.elsevier.com/books/emergency-radiologythe-requisites/ soto/978-0-323-37640-2

20. Kahraman H, Ozaydin M, Varol E et al. (2006): The diameters of the aorta and its major branches in patients with isolated coronary artery ectasia. Tex Heart Inst J., 33(4):463-8.

21. Benedetti N, Hope M (2015): Prevalence and significance of incidentally noted dilation of the ascending aorta on routine chest computed tomography in older patients. J Comput Assist Tomogr., 39(1):109-11.

22. Hatemi AC, Tongut A, Özyedek $\mathrm{Z}$ et al. (2016): Association between ascending aortic diameter and coronary artery dilation: a demographic data analysis. J Int Med Res., 44(6):1349-1358.

23. Triantafyllidi H, Rizos I, Rallidis L et al. (2010): Aortic distensibility associates with increased ascending thoracic aorta diameter and left ventricular diastolic dysfunction in patients with coronary artery ectasia. Heart Vessels, 25(3):187-94.

24. Palomares R, Dux-Santoy L, Guala A et al. (2018): Aortic flow patterns and wall shear stress maps by 4D-flow cardiovascular magnetic resonance in the assessment of aortic dilatation in bicuspid aortic valve disease. Journal of Cardiovascular Magnetic Resonance, 20(1):28-32.

25. Jeudy J, White CS, Kligerman SJ et al. (2018): Spectrum of Coronary Artery Aneurysms: From the Radiologic Pathology Archives. Radio Graphics, 38(1): 11-36.

26. Alegret JM, Calvo N, Ligero C et al. (2010): Dilated aortic root is related to a global aortic dilating diathesis. $\mathrm{J}$ Vasc Surg., 52(4):867-71.

27. Ocak I, Lacomis JM, Deible CR et al. (2011): Prevalence of aortic root dilation in patients with CT angiography of the aorta. Diagn Interv Radiol., 17(3):272-6.

28. Callaghan FM, Bannon P, Barin E et al. (2019): Age related changes of shape and flow dynamics in healthy adult aortas: A 4D flow MRI study. Journal of Magnetic Resonance Imaging, 49(1): 90-100. 